\title{
Community Based Nutrition Programs - Critical design elements and Research needs
}

\author{
EV Shantha ${ }^{1}$, R Shrimpton ${ }^{2}$
}

\section{Introduction}

The nutrition community has been on a learning continuum ever since the first systematic scientific studies on the causes of child malnutrition were reported (Waterlow 1997). Public Health professionals and nutritionists now face newer challenges like the double burden of over and undernutrition (Shrimpton and Rokx 2012) We now know, with a lot more clarity that a life course approach is needed to prevent its consequences (Darnton-Hill et al 2004) and the interventions needed to prevent maternal and child undernutrition during the first thousand days of life starting in uterus (Bhutta, et al 2013). We are less clear on how to tackle the growing problem of overnutrition.

Community Based Nutrition Programs (CBNP) are now reasonably well established as an effective route for bringing about significant reductions in child malnutrition (WHO 2013). There is no single simple definition of CBNP, which can vary from being just the geographic location of the service being delivered, to a situation where service delivery is community driven. The difference being the degree and nature of community participation, and successful CBNPs are ones that move individual and collective dimensions of community participation, from passive receipt to active involvement in the management of services being delivered (Shrimpton 1995). This can be ensured by guaranteeing a combination of contextual (socio-political) programmatic (technical) and financial factors. Evidence demonstrates that with careful design and phased implantation of different components, nutrition activities can enhance positive socio-political factors thereby creating a positive context for nutrition programmes and for capacity building for the broader challenge of social development (Sanders 1999).

We know that it is difficult to replicate the results of small scale efficacy trials into large scale government operated programs however, and this needs a careful crafting of program design, integrating a systemic approach both within and across sectors. Furthermore, increasingly many governments are devolving programming and budgetary responsibilities for social sectors including health to the middle and lower levels of governance. In this context, the importance of sound design and institutional arrangements cannot be overstated (Shrimpton 2002).

This paper deals with design elements of successful CBNPs to effectively reduce child undernutrition. Two important reviews, one by FAO based on the experience gained in nine CBNPs from Africa, Asia and Latin America (Ismail et al 2003) and another by WHO drawing on the results of some 60 projects worldwide (WHO 2013), provide much of the evidence included in this article, but all of which confirms that above all, an enabling environment is necessary to ensure that CBNPs succeed. The focus of this article is not on why government authorities need to intervene to improve maternal and child undernutrition or what interventions are needed, but how to create an enabling environment to ensure that the interventions work at community level in a sustainable manner. The focus is on what we the academic community know about how to do this. The evidence and

\footnotetext{
${ }^{1}$ Development Specialist and Freelance Consultant, Tamil Nadu, India

${ }^{2}$ Adjunct Professor, Department of Global Community Health and Behavioral Sciences, Tulane School of Public Health and Tropical Medicine, New Orleans, USA.
} 
implications for designing successful large scale CBNPs are presented in three sections - The Guiding Principles; The Comprehensive Outcomes; and The Inputs.

\section{The Guiding Principles}

Perhaps the first of the guiding principles in designing CBNP is that it needs a combination of bottom up (horizontal) and top down (vertical) approaches. The term 'community' is quite often used in different contexts to refer to groups of families defined by geographical or local government boundaries. In the context of CBNPs, it is suggested that we use the term to refer to a small group of families where activities take place. It is the level at which decisions regarding infant and young child feeding must be made and responses to childhood illnesses initiated. Documented evidence from many nutrition programs have shown that the top down approach in which a set of activities are handed down to be implemented does not produce sustainable results (Ismael et al 2003). It triggers only a weak response from communities that are supposed to participate. At the same time, communities, nutrition volunteers/workers as well as front line health workers need a lot of capacity building (described more in detail in a later section) and infrastructure support, all of which needs resource allocation not under the control of a small set of families. Needs-based and evidence-based support from top layers of program implementation must be synchronised with active participation by families and communities in decision making and subsequent action to break the cycle of child undernutrition and ill health. The Diagonal approach wherein the horizontal and the vertical approaches move toward each other is obviously the ideal and more sustainable though it takes time to achieve results (Shrimpton 2002).

The second guiding principle is the continuum of community participation. While the obvious for any CBNP is community participation, programs often fail to differentiate between passive vs active participation. Many nutrition programs consider attendance at a weighing session as community participation. Nothing is wrong with this if it is considered as a first step in the continuum of community participation and there are other activities that take the families to the next level of learning, to be able to make the choices for improved child care and towards the desired outcome. But if community participation starts and stops with attendance in weighing or counselling sessions, then sustainable outcomes will never be achieved, as has been demonstrated in the Posyandu in Indonesia (Nazri et al 2016).

The platform or the routes through which communities participate is also important for the outcome. The CBNP should provide opportunities for communities to learn together in smaller groups for e.g. food or cooking demonstrations, as practiced in the Tamil Nadu Nutrition Program (TINP), the Bangladesh Integrated Nutrition Project (BINP), and the Ethiopian CBNP. Sharing experiences with other communities can also facilitate learning in the context of other sectoral inputs like the child (health) days in Ethiopia, learning opportunities in health facilities, through radio programs, etc. Such opportunities for participatory learning help to move the communities in incremental steps in the continuum of participation.

The methodology used in community participation exercises is equally important and should target the affective domain of learning for the communities. Providing information will raise some awareness but often alone will not result in increased participation or learning that will change behaviour or a practice. But it is useful in large settings. This has to be followed up with other guided learning opportunities. Many nutrition programs such as the SECALINE program in Madagascar (Ismael et al 2003) develop a communication strategy based on formative research to support this participation and learning at community level and different layers of program implementation. 


\section{The Comprehensive Outcomes}

A project or a program can only be as good as its stated objective. Hence it is important to have clarity on the various outcomes that CBNP should aim to achieve. Often in the process of designing a program we get bogged down by terminologies relating to goal, impact, outcomes, inputs and outputs. Programs usually have a statement on a long-term goal, as well as specific impact and outcome objectives. In this context, it is useful to refer to Amartya Sen's distinction between "culminating"outcomes and "comprehensive" outcomes (Sen 2000). Culminating outcomes are those where the objective is achieved, regardless of the process, i.e. winning at any cost.

Comprehensive outcomes consider the process of reaching an objective, through dialogue and persuasion, not by imposition. This concept and its relationship to horizontal, vertical and diagonal approaches to nutrition programming is described Figure 1 (Shrimpton 2002). The positive outcome can be something like preventing immune preventable diseases by vaccination which can be achieved as a culminating outcome by rounding up mothers and small children and vaccinating en mass, as is sometimes done in child health days. An alternative way is to get the community to persuade young mothers to bring to bring their children to the health centres regularly and get their vaccinations as part of routine health care, and get other advice and health care as appropriate. This should include being weighed and tracking the growth of the child on the growth chart, the promotion of exclusive breastfeeding and counselling on appropriate complementary feeding. This is a comprehensive outcome.

\section{FIGURE 1-HORIZONTAL, VERTICAL, AND DIAGONAL APPROACHES FOR NUTRITIONAL PROGRAMMING}

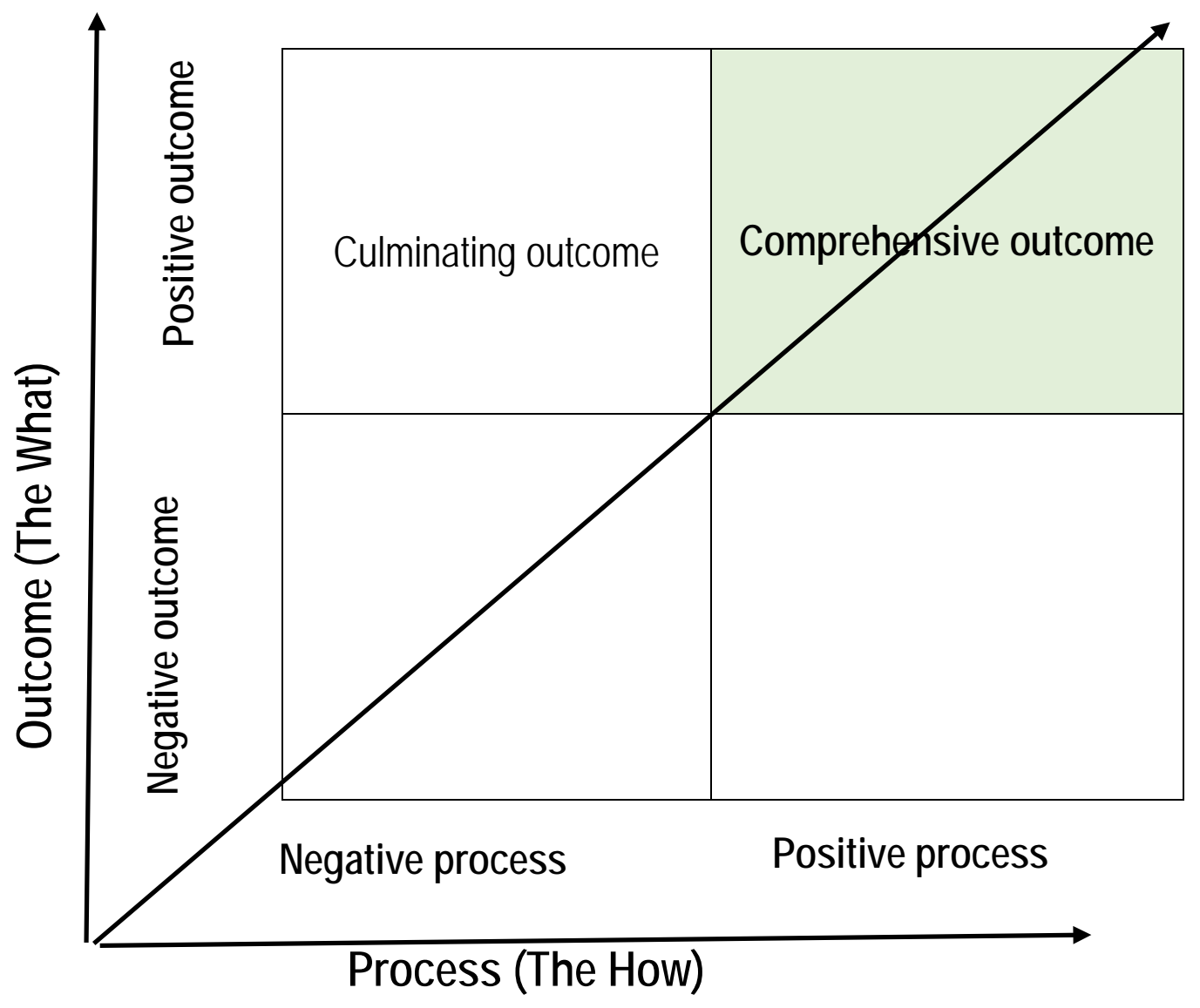


In the context of CBNP, it is important for program managers to focus on how communities/families participate in making the right choices each time, thus adding the incremental learning for everybody that will make the outcome sustainable. Nutrition education should be an instrument of empowerment (Kent 1988) and exercising freedom of choice is an important part of the learning process in reaching the goal in development. As facilitators of development, it is the responsibility of programme managers to provide the appropriate environment for this to happen.

While perfectly designed large scale CBNPs are rare, some programs have at least a few of the elements discussed above (WHO 2013). The Ethiopia CBNP within the National Nutrition Program serves as a reference for clearly defined comprehensive outcomes, including both impact and process outcomes (White and Mason 2012). Another example of a large-scale community based health and nutrition programme is the Family Health Programme in Brazil (Harris and Haines 2010), which began as part of the healthcare reform in Brazil in the 1988 through UNICEF supported primary health care programmes in the northeastern states of Brazil. This involved teams of community health workers promoting growth monitoring, oral rehydration, breastfeeding and immunization (GOBI) and has grown from 2000 teams including 60.000 community health agents in 1998 to 39000 teams incorporating 265,000 community health agents serving 120 million people across all of Brazil in 2014, which is $62 \%$ of the population (Mackinko and Harris 2015). The Family Health Program is targeted at the poorest communities and was shown to have had an important effect on reducing infant mortality in Brazilian municipalities from 1996 to 2004 (Aquino et al 2009).

\section{The Plausibility Approach and Causal Pathways}

Striving to achieve comprehensive outcomes will help us move towards the much desired plausibility approach in public health policy and decision making. The plausibility approach is critical for overall program design. Thinking through causal pathways while planning for CBNP is smart planning. This involves moving beyond the use of UNICEF's conceptual framework [ref] (UNICEF 1990) usually used in programs/projects to tackle child undernutrition. We need to integrate the concepts of the diagonal approach and comprehensive outcomes. This will result in more process-oriented causal pathway diagrams. This has to be adapted to various levels of implementation. This process must be guided to avoid the pitfall of too many causal pathways floating around in the program leading to lack of clear accountability.

Evidence based information is necessary for moving on the right trajectory throughout program implementation. The importance of community based and district based monitoring systems for regular checking on program performance cannot be emphasized enough. In the Indonesian Posyandu the office of the local village head has a board with the "SKDN". These are the initials in Indonesian for the number of children in the district (Sasaran), how many had growth charts (Kartu), how many came to the last Posyandu weighing session (Datang), and how many had grown (Naik) (Lemeina 1989).

The rate of reduction in child undernutrition among the participants compared to the general population is now widely accepted as an impact measure for nutrition programs with no need for special control groups or randomization. Programs achieving a reduction of at least 2 percentage points net a year are considered successful as this represents a rate higher than development alone tends to achieve (Shrimpton, 2002, WHO 2013) and provides an excellent reference point for what we need to know on measuring effectiveness of large scale nutrition programs. The Ethiopian CBNP reported a reduction of -4.3 and $-5.3 \mathrm{ppt} / \mathrm{year}$ which was an additional 3-4 ppt/yr. over the longterm trend in the population as a whole (White and Mason, 2012). Most programs do see a rapid 
rate of improvement initially and this tends to somewhat slow down and stabilize at lower rate of reduction.

\section{The Inputs}

With the leadership provided by a large number of international institutions and aid agencies, we do have many health initiatives that have helped countries to operationalise various programs aimed at improving child survival, protection, care and development. These inputs place the countries in a better position than ever before to tackle the problem of child undernutrition. In the last decade, there has been a quantum jump in training and communication support materials - both generic and country/culture specific. In this section, we will discuss some of the key inputs that can make CBNP successful and sustainable.

\section{Community Health and Nutrition Workers}

The core strength of CBNP is a team of grassroots level workers. Community based health and nutrition workers along with their next level supervisors form the most important part of human resource input in CBNPs. Depending on location we need to differentiate between the health extension worker (HEW) (different countries have different nomenclature) and the community nutrition worker (CNW). The HEWs are often part time casually paid workers linked to the local health facility of the Health Ministry. Evidence suggests there is the potential for great synergy through a combination of the paid and volunteer models (Cherrington et al 2010).

The CNWs to whom we are referring here typically cover a small number of families and are usually volunteers from the community. The CNW's core activities include the following:

- Monthly weighing of the target group of children (usually under 2 years)

- Counselling of mothers and families, especially on breastfeeding and complementary feeding

- Community Conversations based on the situation of young children

- Home visits to follow up on children not coming to the monthly weighing session and/or showing growth faltering

- Referral of sick children or children becoming malnourished to the nearest health facility

In a typical CBNP, all activities are built around the community that comes together to do repeated Assessment, Analysis and Action (triple A cycles) perhaps once a month, making incremental learning possible with each cycle. As a small set of 10-20 families may not have easy access to common property resources, larger community gatherings can take place at the next higher level, such as the village hall or the health post, and can take place less frequently, perhaps once a quarter or every six months. There is an interesting correlation between program intensity (measured by ratio of CNW to families and program impact (sustained reduction in child underweight (ppt/yr) which has been presented in a WHO (2013) publication on Essential Nutrition Action (Figure 2). 
Figure 2. Sustained rate of underweight reduction (ppts/yr) compared to programme intensity, estimated as CHNWs per 1000 children, as part-time equivalents. (WHO 2013; shared with

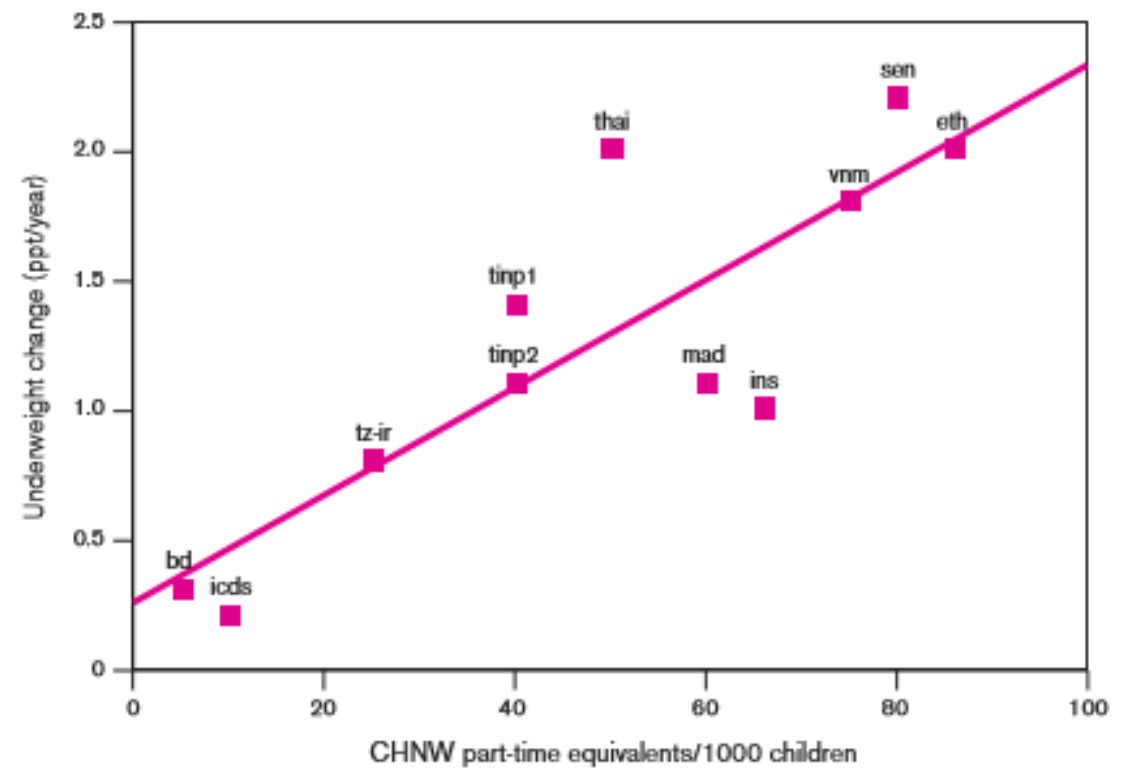

permission)

How manageable the workload of CNWs is, given that few programs can afford to have paid full-time workers at that level, needs attention. By having a higher number of CNWs in the community, we create a critical mass of trained child care workers who have the potential to be change agents. The program will also be able to reach remote areas more effectively when there is one volunteer worker to a small set of 10-20 families. In the case of India's Integrated Child Development Services (ICDS), a paid worker is also expected to conduct pre-school activities for children 3 to $5+$ years, and this workload is often cited as one of the reasons for the low impact on reduction of child undernutrition (Kapil et al 2000).

When the CNWs have a manageable workload, the contact with the families will be more frequent and consistent. An example is the community based growth monitoring and promotion program in Honduras where $83 \%$ of children enrolled were weighed regularly compared to only $70 \%$ in facilitybased programs. With regular follow up visits from the workers and participation in other services, the children in the CBNP had significantly higher weight gain than those in facility based programmes. (Mason et al 2006). The flip side of having voluntary workers is attrition and the need to keep training new recruits. Availability of suitable candidates to take up the work of CNWs is one hurdle projects will face when literacy rates are very low. Training and refresher training content and methodologies have to be suitably adjusted.

Eventually a range of activities relating to health and nutrition will be added to the responsibilities of CNWs especially when this becomes a successful strategy. This is desirable not just for effectiveness but also for organizational efficiency (Mason et al 2006). At the same time, loading CNWs with too many responsibilities will defeat the spirit of volunteerism and divert attention from CBNP objectives (Reis et al 1990). We need to strike a balance. A good strategy will be to set achievable process related outputs as a first goal and add newer activities incrementally over a timeframe. A suitable mix of community based and facility based activities will also increase effectiveness of interventions.

First level supportive supervision is just as important. For CNWs it may be done by HEWs (Ethiopia) or other specially assigned and trained supervisors (TINP, ICDS). Some programs also use NGO staff if they have been given implementation/training roles (BINP). The supervisor/CNW ratio has to be low 
to make supervision and on the job training effective. The best ratio would be one supervisor to 10 to 20 CNWs as per figure 3 (Mason et al 2006).

Figure 3. The general structure of Community Based Nutrition Programmes based on Thailand's Programme (Mason et al 2006; used with permission)

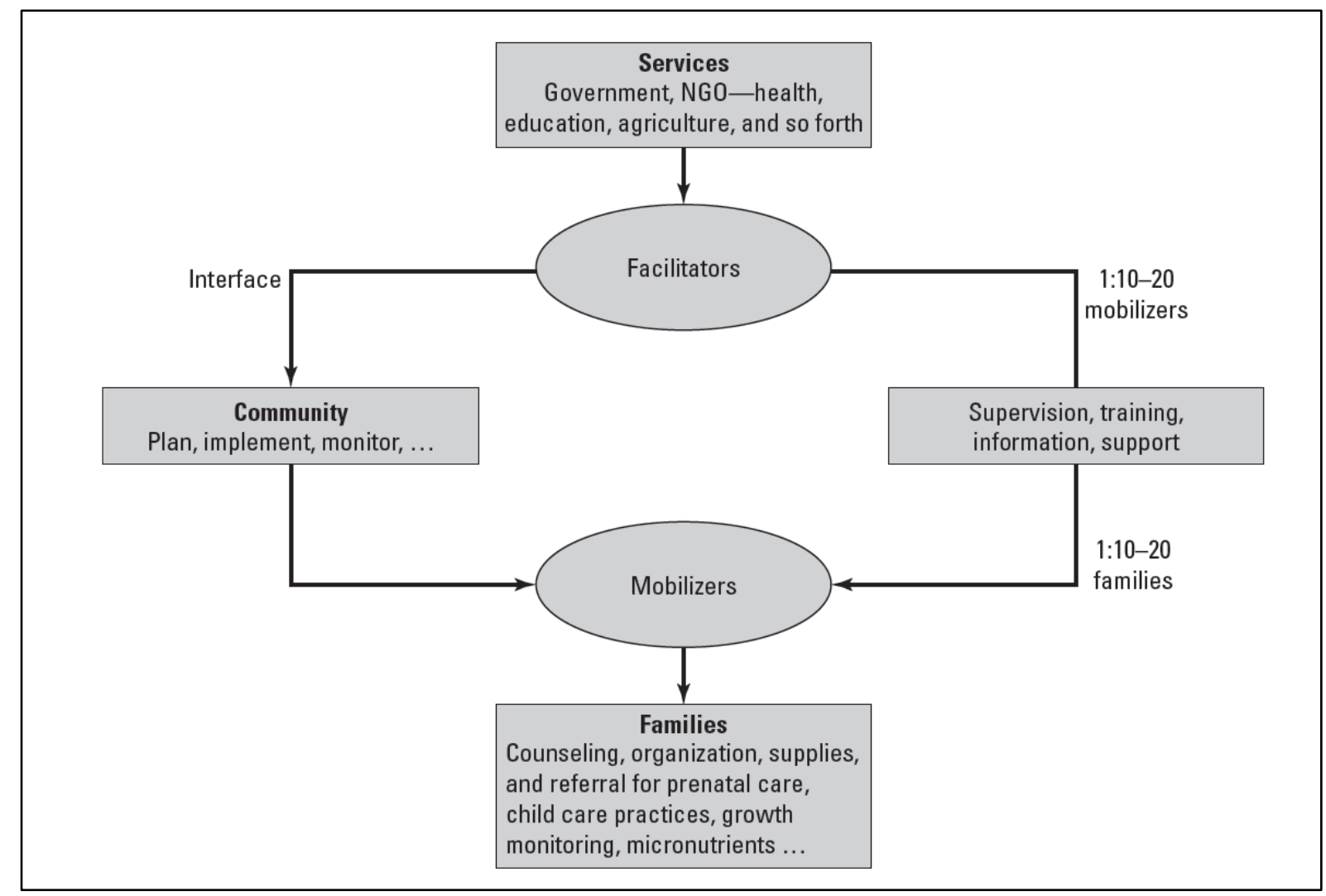

If the supervisor is not just for CBNP and has other responsibilities, it is even more important that the supervisory workload is carefully assessed and training includes guidance on work routines. The most demanding of the supervisory tasks in CBNP relate to data collection, recording and reporting. If technology can be used for more accurate, speedy data collection and reporting, supervisors may be able to improve quality of CBNP. Another important supervisory task is learning to ask and listen before telling or giving instructions. This ability to dialogue is important for counselling, for helping the community in the triple A cycle, and for getting families to participate truly in their development.

\section{Capacity Building and Training at various levels}

It is important to differentiate between capacity building and training. Capacity building empowers individuals and institutions. If our objective is the comprehensive outcomes as shown in Figure 1, then, we need to look at the broader canvas of enhancing the ability of all stakeholders to understand the value based approach to community participation. The four dimensions to capacity building are: Adequate number of nutrition/health managers; appropriate competencies; management support systems; and an enabling working environment (WHO 2007). While all of these are important, we will elaborate on the dimension of competencies for CBNP implementation.

Unlike during the early decades of nutrition programming, a number of programming tools as well as detailed training manuals have been developed and widely used in many countries. These include, among others, IMCl and Community IMCl guidelines, Essential Nutrition Actions (ENA) as recommended by WHO, Infant and young child feeding guidelines, as well as protocols for the 
management of severely malnourished children in the community. Even with these developments, training can be very challenging when the demands for program coverage far exceed the capacity to roll out quality training. A very fast expansion of program area often throws training plans and schedules into disarray, affecting training effectiveness.

CBNPs require intense process-oriented training that not only provides information (knowledge) but also develops the skills and attitudes needed to bring about behaviour changes. A system approach to training starts from needs assessment. Based on a task analysis for the grass roots workers and community level trainers, and some sounding of what CNWs already know, the content and methodology of training are to be worked out. The duration of the training is often a balance between the ideal and the practical. Grassroots level training may last for one month or less than a week. To draw once again on the experience of the Ethiopian CBN, all training modules were developed in partnership with NGOs before training was rolled out at district level.

A number participatory tools like mapping of the area have been used at community level for the identification of households with children, children's health ranking, and understanding child undernutrition in simple terms, as well as the wealth ranking that is usually carried out. In fact, the selection of CNWs by the community should be done only after the initial triple A cycle at the community level and a demand for starting of activities has been generated. While it is not possible to train a CNW in participatory methodologies and individual/family counselling skills in less than a week, it is also not practical to have CNWs undergo long training programs. It is also important to train the first level supervisors who can support a set of CNWs for community level activities and also continuously train CNWs for individual and family counselling.

Thus, training cannot be a one-time activity and in addition, refresher/re-training can provide new information and improve skills. Most projects aim at least for yearly training or more often if possible. Monitoring data and evaluation studies normally provide inputs for refresher training content and methodologies.

\section{Role of NGOs/Civil Society Organizations}

Large scale operational programs have to be based in government departments for long term sustainability. At the same time, government departments in many developing countries do not have sufficient human or financial resources to implement all aspects of a CBNP within a short span of time. The collaborative role NGOs and civil society organizations--including teaching institutions--can play becomes important in this context.

As pointed out earlier, the process-oriented approach in training and community interaction is a prerequisite for achieving sustainable comprehensive outcomes. Technical capacity to undertake this in a large scale can be supported by NGOs. At the same time, coordinating different NGOs and organizations carrying out training activities is often a formidable task for the implementing ministry. There has to be clarity on the role of all partners. Terms of reference (TORs), job descriptions and responsibilities as well as contractual terms should reflect this. NGOs were used in Ethiopia CBN for quickly cascading training and covering large program areas over a short time span. NGOs or nonprofit organizations have also been running the grass roots level and middle level training in the Indian ICDS program in some areas.

\section{Many sectors that have a nutrition responsibility}

Nutrition is most often readily linked to the health and food security sectors, but many other sectors are also critical for sustainable and comprehensive outcomes. Nutrition-sensitive interventions and 
programmes in agriculture, social safety nets, early child development, and education have enormous potential to enhance the scale and effectiveness of national nutrition programmes. Furthermore, improving nutrition can also help nutrition-sensitive programmes achieve their own goals (Ruel et al 2013). At a central level of planning, these sectors must adopt a nutrition sensitive approach and goals. At the middle level, coordination of the different sectors poses challenges but these can be overcome with appropriate capacity building. Some of the strategies that have been tried out effectively for middle level inter-sector coordination include joint planning and review meetings, joint work routines, joint supervision, as well as joint budget preparation.

One important question is, can CBNPs work everywhere and anywhere? There are pre-requisites for an enabling environment and if we can provide the right one, there are the dividends in terms of ensuring a healthier child population. White and Mason (2012) list the contexts in which CBPs can work. This includes a fairly well-developed health extension program that recognizes that acute food insecurity is not the sole or primary cause of malnutrition, as well as the presence of institutions and agencies that can support capacity building and training and monitoring and evaluation. The most important component of an enabling environment is a government that gives the task of reducing child undernutrition a top priority.

\section{The key points to remember for a sustainable CBNP are presented in Box 1.}

Box 1. Dos and don'ts in designing CBNP

- Use both a vertical and horizontal approach - a diagonal approach is desirable

- Communities/participating families must be facilitated to help them make sensible choices so that they can truly participate in their development. Hence CBN is not a fashion word but a most serious and demanding approach to comprehensive outcomes.

- Plan smart. Clearly identify measurable outcomes. Have causal pathways developed for various layers of implementation with accountability for the processes.

- Have an evaluation plan that will stand universal scrutiny. Sometimes faulty evaluations will take away the credit of your achievement.

- Understand the difference between a community based approach and facility based approach and hence the need for program intensity in terms of number of households per CNW - $10-20$ households per CNW.

- $\quad$ First level supervisor ratio to CNWs is equally important and ideal being $1:<20$.

- Training in participatory methodologies and counselling forms the core of a task based training strategy focussing on skills, attitudes and behaviour rather than on knowledge alone.

- Plan for reasonable pace of program expansion covering the population. If it is too slow, impact objectives will not be met. If it is too fast, the quality of training and implementation will be adversely affected.

- No one sector can be responsible for the reduction in child undernutrition. Nutrition sensitive planning and goals must be introduced in many sectors that impact on the underlying causes for malnutrition.

- Roles of NGOs, civil society organizations and the private sector are very important. We will be stronger together and we stand a better chance of winning the war against child undernutrition.

In conclusion, it is often said that nutrition, especially child nutrition, is the story of Everybody, Somebody, Anybody and Nobody. There was an important job to be done and Everybody was sure that Somebody would do it. Anybody could have done it, but Nobody did it. Somebody got angry about that, because it was Everybody's job. Everybody thought Anybody could do it, but Nobody realized that Everybody wouldn't do it. It ended up that everybody blamed somebody when nobody did what anybody could have done. But CBN programming offers the best opportunity to truly make it everybody's responsibility towards our children's future and to deliver on that promise. 


\section{References}

Aquino R, de Oliveira NF, Barreto ML. Impact of the Family Health Program on Infant Mortality in Brazilian Municipalities. American Journal of Public Health. 2009; 99 (1): 87-93

Bhutta ZA, Das JK, Rizvi A, Gaffey MF, Walker N, Horton S, Webb P, Lartey A, Black RE; Lancet Nutrition Interventions Review Group.; Maternal and Child Nutrition Study Group. Evidence-based interventions for improvement of maternal and child nutrition: what can be done and at what cost? Lancet. 2013;382(9890):452-77

Cherrington A, Ayala GX, Elder JP, Arredondo EM, Fouad M, Scarinci I. Recognising the diverse roles of community health workers in the elimination of health disparities: From paid staff to volunteers. Ethn Dis 2010: 20(2):189-194

Darnton-Hill I, Nishida C, James WP. A life course approach to diet, nutrition and the prevention of chronic diseases. Public Health Nutr. 2004;7(1A):101-21

Harris M, Haines A. Brazil's Family Health Programme. BMJ. 2010; 341:c4945

Kapil U, Pradhan R. Integrated child development services scheme (ICDS) in India: its activities, present status and future strategy to reduce malnutrition. J Indian Med Assoc. 2000. 98(9):559-66.

Kent G. Nutrition education is an instrument of empowerment. Journal of Nutrition Education. 1988. 20 (4):193-195.

Leimena SL. Posyandu: A Community Based Vehicle to Improve Child Survival and Development. Asia Pac J Public Health. 1989; 3(4):264-7.

Mason JB, Sanders D, Musgrove P, Soekirman, and Galloway R. Community Health and Nutrition Programmes. Chapter 56 in Disease Control Priorities in Developing Countries, Jamison DT, et al (Eds). 2006. New York: Oxford University Press.

Macinko J, Harris MJ. Brazils family health strategy - delivering community-based primary care in a universal health system. New Eng J Med. 2015. 372 (23): 2177-2181

Nazri C, Yamazaki C, Kameo S, Herawati DM, Sekarwana N, Raksanagara A, Koyama H. Factors influencing mother's participation in Posyandu for improving nutritional status of children under-five in Aceh Utara district, Aceh province, Indonesia. BMC Public Health. 2016. 16:69.

Reis T, Elder J, Satoto J, Kodyat B, Palmer A. An Examination of the Performance and Motivation of Indonesian Village Health Volunteers. International Quarterly of Community Health Education. 1990. 11 (1): $19-27$

Ruel MT, Alderman H, and the Maternal and Child Nutrition Study Group. 2013. Nutrition-sensitive interventions and programmes: how can they help to accelerate progress in improving maternal and child nutrition? Lancet. 382: 536-51

Sanders D. Success factors in community-based nutrition programmes. Food and Nutrition Bulletin. 1999. 20(3): 307-314

Sen A. 2000. Consequential evaluation and practical reason. The Journal of Philosophy XCVII (9): 477502

Shrimpton, R. "Community Participation in Food and Nutrition Programs: An Analysis of Governmental Experiences". In: Child Growth and Nutrition in Developing Countries: Priorities for 
action, pp 243 261. Pinstrup-Andersen, P, Pelletier, D, Alderman, H.(Eds). 1999. Ithaca: Cornell University Press.

Shrimpton R. "Nutrition and Communities." In Nutrition: A Foundation for Development. 2002 Geneva: ACC/SCN.

Shrimpton R and Rokx C. The Double Burden of Malnutrition: A review of global evidence. Health Nutrition and Population (HNP) Discussion Paper. 2012. Washington DC: The World Bank.

UNICEF. Strategy for improved nutrition of children and women in developing countries. New York: United Nations Children Fund. 1990.

Victora CG, Habicht JP, Bryce J. Evidence-based public health: moving beyond randomized trials. Am J Public Health.2004. 94 (3):400-5.

Waterlow JC. Protein-energy malnutrition: the nature and extent of the problem. Clin Nutr. 1997 Mar;16 Suppl 1:3-9.

White J and Mason J. Assessing the impact on child nutrition of the Ethiopia Community-based Nutrition Program. Report to UNICEF of an evaluation study. 2012. New Orleans: Tulane University School of Public Health and Tropical Medicine.

WHO. Towards better leadership and management in health: Report of an international consultation on strengthening leadership and management in low-income countries. MAKING HEALTH SYSTEMS WORK: WORKING PAPER No. 10. WHO/HSS/healthsystems/2007.3. Geneva: World Health Organization.

WHO. Essential Nutrition Actions: Improving maternal, newborn, infant and young child health and nutrition. 2013. Geneva: World Health Organization. 